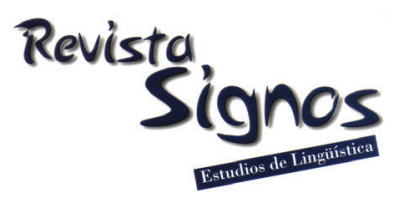

\title{
La influencia del área disciplinar en la variación terminológica: Un estudio en un corpus interdisciplinario sobre pesca*
}

\section{The influence of the disciplinary field on terminological variation: $A$ corpus-based study in the interdisciplinary domain of fishing}

\author{
Sabela Fernández Silva \\ Pontificia Universidad Católica de Valparaíso \\ CHILE \\ sabela.fernandez@ucv.cl
}

Recibido: 9-X-2012 / Aceptado: 31-V-2013

\section{Resumen}

La división de la ciencia en disciplinas se ha justificado, tradicionalmente, porque cada disciplina observa un segmento de la realidad desde una perspectiva particular, obteniendo un sistema conceptual y una terminología propios. Sin embargo, la ciencia ha avanzado enormemente gracias al trabajo interdisciplinar y muchos de los avances científicos de las últimas décadas se han forjado con la combinación de teorías, métodos y conceptos de diferentes disciplinas. En este contexto interdisciplinar, en que el conocimiento se construye y se comparte entre especialistas de diferentes áreas, nos hemos preguntado de qué manera se reflejan las diferencias disciplinares en el uso de la terminología. Para ello, analizamos los temas, conceptos y variantes denominativas en un corpus bilingüe (francés y gallego) de textos sobre pesca escritos por expertos en biología, economía y derecho. Un análisis semántico-cognitivo de las variantes denominativas nos permitió describir diferencias de perspectiva en las denominaciones empleadas por los tres colectivos para referirse a conceptos de la pesca. Los resultados muestran una elevada coincidencia en los temas y conceptos abordados y diferencias entre el subconjunto de términos compartidos por las tres áreas y el subconjunto exclusivo de cada área. Los términos compartidos son más cortos, más frecuentes, neutros en cuanto a la perspectiva y se corresponden con el nivel básico de categorización de la terminología pesquera. El subconjunto no compartido muestra una mayor inestabilidad léxica, mayor variabilidad en cuanto a la estructura morfosintáctica, mayor dependencia del contexto de ocurrencia y refleja perspectivas de conceptualización menos prototípicas de la terminología de la pesca y más vinculadas al área disciplinar.

Palabras Clave: Terminología, área disciplinar, variación denominativa, variación disciplinar, discurso especializado. 


\begin{abstract}
The division of Science into disciplinary fields was traditionally explained by the fact that each discipline observes a segment of reality from a particular perspective. Therefore, each discipline had its own conceptual system and terminology. But Science has also progressed through interdisciplinary work teams, and great scientific advances have been made possible through the combination of theories, methods and concepts from different subject fields. In this context, where knowledge is built and shared by specialists from different disciplinary fields, we asked ourselves how disciplinary differences are reflected in the use of terminology. In order to do so, we analyzed the topics, concepts and term variants in a bilingual French and Galician corpus of texts about fishing, written by biologists, economists and law experts. By describing the conceptual information displayed by term variants, we were able to describe differences in the perspective in the denomination of fishing concepts among the three groups. The results show a high overlap regarding the topics and concepts and differences regarding the subset of term variants shared by the three collectives as compared to the subset of variants exclusive to each group. Shared terms are shorter, more frequent, and more basic to fishing terminology; their perspective regarding the object is neutral. In contrast, however, unshared terms show greater lexical instability, are strongly context dependent and sometimes reflect perspectives of conceptualization that are less prototypical of fishing terminology and more related to the expert's domain of specialization.
\end{abstract}

Key Words: Terminology, subject field, term variation, disciplinary variation, specialized discourse.

\title{
INTRODUCCIÓN
}

La división de la ciencia en disciplinas y subdisciplinas es una práctica aceptada en la ciencia y en los estudios sobre lenguajes de especialidad. Esto se justifica, en los círculos científicos, en el hecho de que cada disciplina se interesa por una parcela de la realidad, desde una perspectiva y con unos objetivos particulares, lo cual resulta en una estructuración conceptual propia. Por otro lado, en la teoría y la práctica terminológica, el área temática o disciplinar es el elemento organizador del conocimiento, y en torno a ella se estructuran los sistemas conceptuales y las terminologías. Sin embargo, la división de la ciencia en disciplinas independientes es problemática y no existe un acuerdo entre las múltiples clasificaciones que se han propuesto. Por otro lado, la ciencia tiene un componente interdisciplinar muy importante y muchos desafíos científicos se han enfrentado exitosamente con la combinación de métodos, conceptos y perspectivas procedentes de distintas disciplinas. Aunque perviva la distinción entre áreas disciplinares, estas no se conciben como entidades monolíticas sino como espacios del saber con zonas comunes, en el que los conceptos surgidos de un área pueden ser apropiados y reinterpretados en otras, conviviendo una pluralidad de perspectivas sobre la misma realidad. 
En este artículo hemos querido explorar esta variabilidad de perspectivas a partir de la variabilidad lingüística, mediante el análisis de la variación terminológica en un área interdisciplinar. Nos hemos preguntado de qué manera conviven estas diferencias disciplinares cuando se aborda una misma temática, y qué reflejo tiene en la terminología utilizada. Para ello, hemos compilado un corpus de textos sobre pesca producidos por expertos en biología, economía y derecho, y hemos comparado los temas, conceptos y las variantes denominativas empleadas por los tres colectivos. A fin de comparar las perspectivas de conceptualización, describimos las variantes a partir de la información conceptual reflejada en la forma denominativa.

El artículo se estructura en los siguientes apartados. En el apartado del marco teórico, exponemos las ideas principales sobre el conocimiento especializado y su división disciplinar, y damos cuenta de estudios previos sobre variación terminológica en relación con el área disciplinar. En el apartado del marco metodológico, presentamos el corpus y la metodología de análisis semántico-cognitivo de las denominaciones. En el apartado de resultados, comparamos en primer lugar el solapamiento temático y conceptual entre los tres subcorpora; en segundo lugar, analizamos el solapamiento denominativo, y comparamos las características de los términos compartidos y de los términos exclusivos de cada área; y, por último, comparamos las perspectivas de conceptualización en las denominaciones que emplea cada colectivo para referirse a conceptos de animales. Finalmente, en el último apartado presentamos las conclusiones de nuestro estudio.

\section{Marco teórico}

\subsection{El conocimiento especializado y la división disciplinar}

Las nociones de conocimiento especializado, conocimiento general, o disciplina son fundamentales en la teoría terminológica y en los estudios sobre lenguajes de especialidad. La idea de que existe un conocimiento especializado, que se obtiene por distintos medios y se estructura de diferente manera que el conocimiento general, es uno de los criterios que se presentan en la bibliografía para diferenciar el lenguaje especializado del lenguaje general y, por lo tanto, la terminología del léxico general (Wüster, 1979; Cabré, 1999a; Temmerman, 2000).

A la hora de caracterizar el conocimiento especializado, a menudo equiparado con el conocimiento científico, todavía pervive una concepción objetivista entre algunos especialistas y filósofos de la ciencia. Así, para Bunge (1966), la ciencia es aquel conocimiento racional, sistemático, verificable y por consiguiente fiable. Sin embargo, otros autores (Chalmers, 1990) cuestionan esta visión monolítica e idealista de la ciencia y defienden la necesidad de tener en cuenta el contexto histórico-social en el que se produce y los objetivos planteados por cada área del conocimiento. 
Para Cabré (1999b), la temática específica es la que permite diferenciar el lenguaje general de los lenguajes de especialidad. Estas temáticas especializadas son ámbitos de contenidos que no forman parte del conocimiento general de los hablantes de una lengua y que son objeto explícito de aprendizaje académico o profesional. Este conocimiento se adquiere "mediante transmisión verbal de los conocimientos por parte de los especialistas en la materia, o de la transmisión de la experiencia por contacto con el medio profesional" (Cabré, 1999b: 175). El conocimiento especializado es fruto de la observación de una determinada parcela de la realidad con una perspectiva particular, impuesta por la especialidad a la que pertenecen:

"Aquesta transmissió no suposa només el traspàs del contingut seqüencial de la matèria, sinó sobretot la perspectiva des de la qual aquesta matèria ha de ser conceptualitzada. És precisament aquesta òptica la que dóna la característica d'especialitzat a un tema determinat. Aprendre, doncs, una temàtica especialitzada significa interíoritzar els seus continguts i l'òptica de la seva significació." (Cabré, 1999b: 175)

La división del conocimiento especializado en áreas disciplinares es consustancial a la actividad científica y a los estudios sobre lenguajes de especialidad. En efecto, la observación de diferentes parcelas de la realidad, con distintas perspectivas, métodos y objetivos, justifica una división natural de los saberes y la conformación de diferentes sistemas de conocimientos. En palabras de Cabré (2008: 20):

“[...] a contextos distintos les corresponden necesidades distintas y las necesidades empujan a los seres humanos a "ver" fenómenos que otros individuos en condiciones distintas ni siquiera perciben. [...] Y es este mismo principio el que nos sirve para explicar la percepción desde las especialidades."

Pero las divisiones entre disciplinas también tienen una justificación social: los miembros que se organizan en torno a una misma actividad científica o técnica y que comparten a través de los mismos circuitos tradiciones, convenciones y prácticas discursivas, desarrollan un sentimiento de pertenencia a una comunidad socioprofesional (Swales, 1990; Gambier, 1991; Hyland, 2000; Hyland \& Bondi, 2006).

En el área de la escritura académica, las Lenguas con Propósitos Específicos y la lingüística textual ha surgido recientemente un interés por caracterizar las diferencias entre disciplinas en cuanto a sus prácticas discursivas (Hyland \& Bondi, 2006). Así, se ha observado que los géneros textuales prototípicos varían en función de la disciplina (Parodi, 2010a), y que géneros textuales transversales a las disciplinas académicas, como los artículos científicos, las tesis o los manuales, también experimentan variaciones en cuanto a su organización retórico-funcional (Samraj, 2005, 2008; Ibáñez, 2010; Parodi, 2010b; Sabaj, 2012).

Pero sin duda, el elemento diferenciador por antonomasia del discurso de cada disciplina es la terminología, pues es a través de estas unidades que se condensa 
el conocimiento especializado en los textos. Para producir o comprender un texto especializado es necesario conocer el significado preciso de los términos y las relaciones que estos mantienen en la estructura conceptual del ámbito. Por ello, en la teoría conceptual terminológica tradicional (Wüster, 1979) se postulaba que los términos solo se podían considerar como tales si se asignaban a un área temática determinada y, como consecuencia, estos eran monosémicos al interior de cada área temática (Tebé, 2005).

A pesar de que el área temática o el área disciplinar es un elemento estructurador de las terminologías, la división entre áreas temáticas no es tan natural, clara y nítida como se consideraba tradicionalmente. Existen importantes parcelas de conocimiento de carácter interdisciplinar, así como una multiplicidad de puntos de vista al interior de una misma disciplina según escuelas de pensamiento, contextos culturales, intereses individuales y colectivos o distintos estadios de desarrollo disciplinar.

En los ámbitos interdisciplinarios, los mismos conceptos son abordados desde distintas disciplinas y esta pluralidad de perspectivas conlleva diferencias en la conceptualización, tal y como señalan numerosos autores (Cabré, 1999a; Gaudin, 2003; Tebé 2005).

Por lo tanto, aunque perviva la distinción entre áreas temáticas, estas no se conciben como entidades independientes sino como un espacio del saber con zonas comunes, en el que los conceptos surgidos en un área pueden ser apropiados y reinterpretados en otras, contribuyendo en conjunto a la evolución del conocimiento. Como expresa acertadamente Tebé (2005: 72):

"L’àrea temàtica, en conseqüència, és un espai viu, en moviment, que redefineix constantment els seus límits, i que pot acceptar diverses interpretacions."

\subsection{La motivación cognitiva, comunicativa y lingüística de la variación denominativa}

La 'variación denominativa' es el fenómeno por el cual a un mismo concepto le corresponden diversas denominaciones. La razón por la que algunos autores (Freixa, 2002; Suárez, 2004; Cabré, 2008; Fernández-Silva, 2011; Seghezzi, 2011) preferimos 'variación denominativa' a sinonimia se debe a la perspectiva onomasiológica adoptada: el concepto, elemento cognitivo, se proyecta a través de unidades terminológicas diferentes en los textos, sin que estas sean necesariamente sinónimas entre sí desde el punto de vista semántico (Fernández-Silva, Cabré \& Freixa, 2012).

Tradicionalmente, se consideraba que la variación denominativa era contraria a los ideales de precisión del lenguaje científico, y su existencia se negaba a nivel teórico y se proscribía en el trabajo terminológico (Wüster, 1979). Si bien en la actualidad todavía existe la percepción de que la variación denominativa es perjudicial, sobre todo en 
los círculos de especialistas, en la teoría terminológica su existencia está plenamente reconocida, y todas las propuestas teóricas actuales reconocen plenamente la variación (Cabré, 1999a; Temmerman, 2000; Gaudin, 2003; Diki-Kidiri, 2008; Faber, 2012).

Las causas de variación denominativa (Fernández-Silva, 2011) se pueden relacionar con la triple naturaleza de la unidad terminológica postulada en la Teoría Comunicativa de la Terminología (Cabré, 1999a):

a) El término es una unidad cognitiva, puesto que representa un concepto especializado. Por lo tanto, la variación es el reflejo lingüístico de la dinamicidad de la categorización, la configuración flexible de los conceptos especializados, y la multiplicidad de puntos de vista sobre la misma realidad que existen entre disciplinas, escuelas de pensamiento, individuos, etc.

b) El término es una unidad comunicativa, y por lo tanto su forma y contenido se adecúan a las circunstancias en que se produce el acto denominativo. En función de los interlocutores, el registro o el canal, se requerirán diferentes términos para hacer referencia a un mismo concepto. Del mismo modo, un mismo autor puede recurrir a la variación denominativa para enfatizar diferentes dimensiones del concepto en diferentes ocasiones, en función del punto de vista que quiere ofrecer (Bowker, 1997; Fernández-Silva, 2013).

c) El término es un signo lingüístico dotado de significante y significado, que remite a un concepto especializado en el plano cognitivo. Por lo tanto, es preciso distinguir entre concepto y significado como dos entidades de contenido de distinta naturaleza (cognitiva y lingüística), y reconocer que la relación entre estos dos elementos no es directa, sino múltiple, y puede dar lugar a variación bajo forma de polisemia y/o sinonimia (Freixa, 2006; Cabré, 2008). Por otro lado, los términos se entretejen entre ellos y con otras unidades lingüísticas en el interior de un texto, constituyendo un recurso cohesivo esencial para dotarlo de textualidad y posibilitar la comprensión del mismo (Halliday \& Hasan, 1976; Collet, 2004; Louwerse, 2004).

\subsection{El área disciplinar como factor de variación terminológica}

La relación entre área disciplinar y variación terminológica, si bien se menciona en la bibliografía (Cabré, 1999a; Temmerman, 2000; Gaudin, 2003; Tebé, 2005), no ha sido investigada en profundidad mediante estudios empíricos. Tradicionalmente, los términos se describían como monosémicos en el interior de un área disciplinar, y su uso en diferentes áreas se describía como un fenómeno de homonimia, y no de polisemia (Wüster, 1979).

La mayoría de los estudios que relacionan variación terminológica y área disciplinar contemplan este fenómeno desde una perspectiva cognitiva, como un factor que determina diferentes maneras de percibir la realidad y, por tanto, diferencias de conceptualización entre colectivos de especialistas. Este factor se ha relacionado sobre 
todo con la polisemia o variación conceptual del término. Por ejemplo, Tebé (2005) analiza cómo un mismo término, cuando es usado en diferentes disciplinas, conserva un núcleo conceptual común y que, por tanto, debería ser tratado como una unidad polisémica y no como términos distintos en los bancos de datos terminológicos. Por otro lado, Bouveret y Gaudin (1997) analizan las diferencias de conceptualización que existen cuando los mismos términos son empleados por biólogos o estadísticos. Por último, en el contexto de la detección automática de terminología, Névéol y Ozdowska (2006) muestran que existe variación denominativa en textos sobre biomedicina, cuando estos son emitidos por médicos o juristas; por lo tanto, se debe tener en cuenta la pluralidad de perspectivas en el enriquecimiento automático de terminologías:

"La prise en compte du spectre complet d’un domaine de spécialité -ici, le domaine médical-à différents niveaux du spectre permet de compléter une terminologie du domaine." (Névéol \& Ozdowska, 2006: 19)

Por otro lado, la existencia de diferentes corrientes o escuelas de pensamiento en el interior de una misma disciplina se menciona en la bibliografía como causa de variación conceptual y denominativa. Tebé (2005: 73) apunta que la segmentación de la realidad en ámbitos puede variar a causa de la "existencia de puntos de vista diferentes según escuelas o corrientes de pensamiento". Bowker (1997) sugiere que la multidimensionalidad de la clasificación conceptual puede estar originada por la presencia de diferentes escuelas de pensamiento; Bowker y Hawkins (2006) comentan que para denominar las enfermedades en medicina diferentes escuelas seleccionan aspectos diferentes de la enfermedad; y Freixa (2002) también observa que con frecuencia cada escuela emplea sus propias denominaciones para referirse a conceptos idénticos o muy próximos, en algunos casos por diferencias de conceptualización, y en otros, por voluntad de diferenciarse de otras escuelas:

"Normalment, aquestes diferències en les denominacions responen a maneres diferents de percebre $i$ representar-se mentalment la matèria i, consegüentment, a la manera de designar-la. D’altres vegades, però, aquesta variació denominativa respon a una voluntat d'un autor, un corrent o una escola de pensament, de separar-se d'altres autors, corrents o escoles que ja han denominat prèviament aquells conceptes." (Freixa, 2002: 155)

Por último, cabe mancionar otros estudios que han abordado la variación terminológica entre grupos profesionales más allá del ámbito estrictamente académico, entre profesionales con diferentes niveles socioeducativos. Por ejemplo, Conceição (2006) analiza las diferencias conceptuales y denominativas entre especialistas universitarios (profesores, técnicos) y profesionales dedicados al marisqueo. Los niveles de conocimiento dispar de la lengua de especialidad y de la lengua general aparecen como factor que provoca la falta de entendimiento entre colectivos: 
“A incompreensão deve-se ao uso de denominações diferentes, para os mesmos referentes, causado por níveis de conhecimentos diferentes e por questões dialectais e culturais.” (Conceição, 2006: 496)

Por otro lado, Bertaccini y Mateucci (2005) analizan la variación terminológica entre ingenieros, técnicos y obreros empleados en una misma fábrica. Los resultados del estudio, realizado a través de encuestas sociolingüísticas, ponen de manifiesto la 'estratificación lingüística del entorno de trabajo' (Bertaccini \& Matteucci, 2005). La elección denominativa varía según el grupo de locutores y, además, en los estratos socioprofesionales más bajos se da una mayor variación denominativa:

"Sur le plan sociolinguistique, par exemple, le choix terminologique est lié au "status socioculturel" des locuteurs. Ils utilisent la langue de manière différente sur la base de leurs connaissances personnelles et professionnelles. Tous les niveaux de la langue peuvent ainsi établir un contact et déterminer des choix terminologiques intéressants." (Bertaccini \& Matteucci, 2005: 4)

\section{Marco metodológico}

En este apartado presentamos la metodología empleada en el presente estudio. En primer lugar presentamos el corpus textual, a continuación los pasos para la obtención del corpus terminológico y, por último, el método de análisis semánticocognitivo que nos permitió comparar la información conceptual expresada en las variantes denominativas.

\subsection{Corpus textual}

El corpus empleado para este estudio está conformado por 41 textos (288.054 palabras) pertenecientes al área de la pesca, escritos en gallego y francés. El corpus fue compilado para un estudio de mayores dimensiones (Fernández-Silva, 2011), en las que se exploraba el rol de la lengua en la variación denominativa, así como otros factores de orden cognitivo, como la clase conceptual y el punto de vista.

Los textos se agrupan en tres áreas disciplinares: biología, economía y derecho. Para identificar el área, se tuvieron en cuenta factores externos al texto, concretamente la especialización de los autores, su afiliación profesional y/o institucional, y el ámbito de circulación de estos (destinatarios potenciales y ámbito temático de la publicación). Posteriormente, se corroboró la correcta asignación del área mediante un análisis del contenido. En la Tabla 1 mostramos el número de textos y palabras disponibles para cada área disciplinar. 
Tabla 1. Distribución de los textos en función del área disciplinar.

\begin{tabular}{|l|c|c|}
\hline Área disciplinar & $\mathbf{N}^{\circ}$ textos & $\mathbf{N}^{\mathbf{o}}$ palabras \\
\hline Biología & 14 & 65.925 \\
\hline Economía & 19 & 188.751 \\
\hline Derecho & 7 & 33.378 \\
\hline Total & 41 & 288.054 \\
\hline
\end{tabular}

\subsection{Corpus terminológico}

A partir del corpus textual se seleccionaron 95 conceptos de la pesca, representados por un total de 946 variantes denominativas. La selección de los conceptos de análisis se basó en tres criterios principales:

d) Pertinencia temática: Se seleccionaron conceptos básicos o centrales del ámbito de la pesca.

e) Frecuencia y distribución homogénea en los textos: Se priorizaron los conceptos con una frecuencia de aparición elevada, y una distribución homogénea en los subcorpus de biología, derecho y economía (frecuencia media de 85 ocurrencias/ concepto).

f) Diversidad denominativa: De los conceptos centrales con representación en los tres subcorpus se retuvieron aquellos con mayor diversidad denominativa, con una media de 9,9 variantes por concepto.

De todas las unidades que representan conocimiento especializado en los textos, ya sean lingüísticas (desde morfemas hasta frases o párrafos completos) o pertenecientes a otros sistemas de representación (imágenes, fórmulas, gráficos, etc.), hemos seleccionado para nuestro análisis únicamente las unidades terminológicas, según la siguiente definición de Cabré y Estopà (2005: 77):

"Unidad terminológica (UT): unidad léxica, cuya estructura corresponde a una unidad léxica de origen o producto de la lexicalización de un sintagma, que posee un significado específico en el ámbito al que se asocia y es necesaria en la estructura conceptual del dominio del que forma parte."

De esta definición, y por tanto de nuestro análisis, fueron excluidas las unidades inferiores o superiores a la unidad léxica, como morfemas, unidades fraseológicas u oraciones. En lo referente a las unidades sintagmáticas, incluimos estructuras complejas (por ej.N+Adj.+Adj.), siempre y cuando tuvieran estabilidad denominativa, autonomía discursiva, y fueran validados por expertos del área como términos propios de la especialidad. ${ }^{i}$ El proceso de validación se secuenció en las siguientes etapas: 
1) Se proporcionó a los especialistas una lista con los grupos polidenominativos -las variantes agrupadas por concepto-.

2) Se pidió que señalaran los conceptos y/o términos que no consideraban propios de su ámbito.

3) Se pidió que confirmaran la sinonimia entre las variantes de cada grupo polidenominativo. Para ello, se les dio una lista con los términos fuera de contexto y, a continuación, otra con los mismos términos en contexto.

4) Una vez validada la lista de variantes para cada lengua, se pidió al especialista con competencia bilingüe que confirmara la equivalencia entre lenguas, y en caso de no darse una equivalencia total, que indicara en qué aspectos diferían.

Las entrevistas mantenidas con ellos han servido para dotar de fiabilidad al material de análisis, pero también para acceder a una comprensión más afinada del sistema conceptual de la pesca y familiarizarnos con la realidad del sector pesquero en Galicia, Francia y Europa. Por otro lado, la experiencia de trabajo con diferentes especialistas ha puesto de manifiesto el grado de subjetividad inevitable que implica la detección de la sinonimia en corpus. La actitud positiva o negativa del evaluador hacia la variación influye su identificación en discurso y esta actitud, a su vez, viene determinada por el bagaje lingüístico y profesional. Así, el especialista en legislación pesquera, ha sido mucho menos tolerante hacia la variación y encontraba una especificación semántica en cada término; sin embargo, el especialista en ingeniería aceptaba con mayor facilidad la variación.

\subsection{Análisis semántico-cognitivo: Patrones conceptuales de variación denominativa}

Una vez conformados los grupos polidenominativos (conjunto de variantes denominativas que hacen referencia a un mismo concepto), llevamos a cabo un análisis semántico-cognitivo de la información conceptual expresada en las variantes (Kageura, 2002; Fernández-Silva, Freixa \& Cabré, 2011). Tomamos como premisa el carácter motivado de la formación terminológica (Kocourek, 1991), vale decir, que la forma del término guarda una relación con el concepto porque refleja parte de su contenido. Los términos fueron descritos como combinaciones de conceptos, representados por sus constituyentes, dentro del sistema conceptual del ámbito. El núcleo representaría la clase conceptual a la que pertenece el concepto, y el modificador la(s) característica(s) distintiva(s). Por lo tanto, los patrones conceptuales de cada 
variante se obtendrían clasificando la naturaleza conceptual de los constituyentes y de las relaciones entre ellos (ver Figura 1):

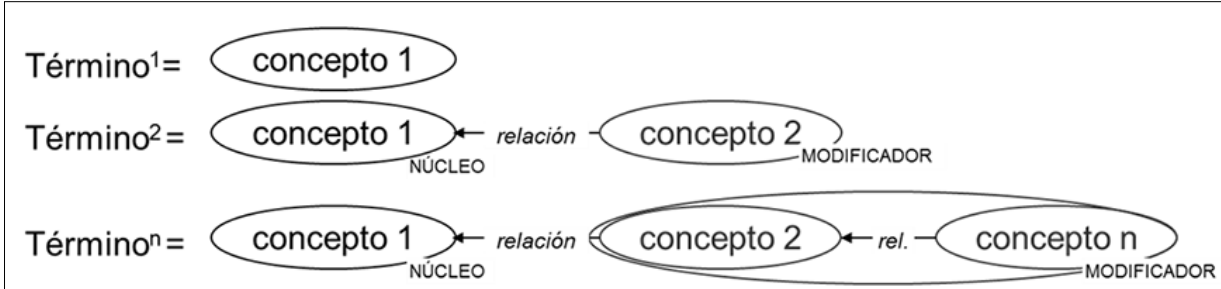

Figura 1. Obtención de los patrones conceptuales de formación terminológica.

En la Tabla 2 mostramos la aplicación del análisis a las variantes denominativas. Tras segmentar las unidades en constituyentes e identificar su posición de núcleo o modificador, se procede a identificar la clase conceptual. En los siguientes ejemplos, los núcleos representan conceptos de grupos de animales (espèce) o de objetos ('producto' y 'recurso'); por su parte, dentro de la clase de objetos, 'producto' y 'recurso' se diferenciarían porque el primero enfatiza la actividad realizada (producir) y el segundo la función para el hombre (abastecerse).

Tabla 2. Identificación de los patrones conceptuales de las variantes denominativas.

\begin{tabular}{|c|c|c|c|c|c|}
\hline Información lingüística & \multicolumn{4}{|c|}{ Patrón conceptual } \\
\hline Núcleo & Modificador & Núcleo & Relación & Modificador \\
\hline Espèce & exploitée & $\begin{array}{c}\text { GRUPO ANIMALES (POR } \\
\text { CARACT. COMUNES) }\end{array}$ & USO DIRECTO & PRODUCCIÓN GENERAL \\
\hline Producto & mariño & $\begin{array}{c}\text { OBJETO GENERAL (POR } \\
\text { ACTIVIDAD) }\end{array}$ & UBICACIÓN & LUGAR GEOGR. (POR PRESENCIA DE \\
\hline Recurso & $\begin{array}{c}\text { pesqueiro e maris- } \\
\text { queiro }\end{array}$ & $\begin{array}{c}\text { OBJETO GENERAL (POR } \\
\text { FUNCIÓN) }\end{array}$ & $\begin{array}{c}\text { USO DIRECTO } \\
+ \text { YUXTA- } \\
\text { POSICIÓN }\end{array}$ & $\begin{array}{c}\text { PRODUCCIÓN } \\
\text { ANIMAL (POR } \\
\text { TIPO DE ACTIVI- } \\
\text { DAD) }\end{array}$ & $\begin{array}{c}\text { PRODUCCIÓN ANI- } \\
\text { MAOR TIPO DE }\end{array}$ \\
\hline
\end{tabular}

En cuanto a los modificadores, el constituyente mariño representa concepto de lugar geográfico (definido por la presencia de agua), y los otros modificadores conceptos de actividades de producción general ('explotación') o de producción animal, caracterizada ésta a partir de la actividad específica (pesca, en pesqueiro) o del objeto de la actividad (marisco, en marisqueiro). Por último, mediante un análisis de las relaciones de dependencia entre los conceptos representados por los constituyentes, se identifica la relación intraconceptual. Retomando el ejemplo, la relación existente en la variante producto mariño sería la de ubicación -el modificador especifica la ubicación del concepto del núcleo- y en las variantes espèce exploitée y recurso pesqueiro la relación principal sería la de uso directo -el modificador expresa el uso al que está destinado el objeto representado en el núcleo. Por último, en el modificador complejo pesqueiro e marisqueiro se identificaría la relación secundaria de yuxtaposición. 
Este tipo de análisis presenta dos ventajas fundamentales para nuestro estudio. En primer lugar, el hecho de analizar la información conceptual como combinación de conceptos en el interior de una estructura conceptual organizada jerárquicamente permite agrupar los patrones conceptuales en grupos y relacionar mejor la información conceptual. Por otro lado, a lincluir como último criterio de clasificación la característica distintiva expresada en la denominación, se logra el nivel de especificidad necesario para comparar las diferentes perspectivas en la conceptualización (y denominación) del concepto en cuestión.

\section{Resultados}

En este apartado presentamos los resultados del estudio en el siguiente orden: En primer lugar, examinamos el solapamiento temático y conceptual entre los subcorpus de biología, economía y derecho; en segundo lugar, el solapamiento denominativo, a saber, la proporción y características del conjunto de variantes comunes a los tres subcorpus y del subconjunto de variantes exclusivas de cada subcorpus. Por último, comparamos cuantitativamente las perspectivas reflejadas en las denominaciones de los conceptos de animales entre los tres subcorpus.

\subsection{Solapamiento temático y conceptual}

En primer lugar, hemos comparado los temas y los conceptos tratados en los textos de biología, economía y derecho, con el objetivo de analizar qué aspectos de la actividad pesquera interesan más a los distintos colectivos, cuáles son los temas comunes y desde qué perspectiva son abordados.

En la Figura 2 representamos el grado de solapamiento temático entre áreas. Podemos ver que existen temas centrales que interesan a las tres disciplinas (como la producción o la gestión de pesquerías) y otros que se vinculan más naturalmente a un área concreta (como el empleo en economía o los ecosistemas costeros en biología). 


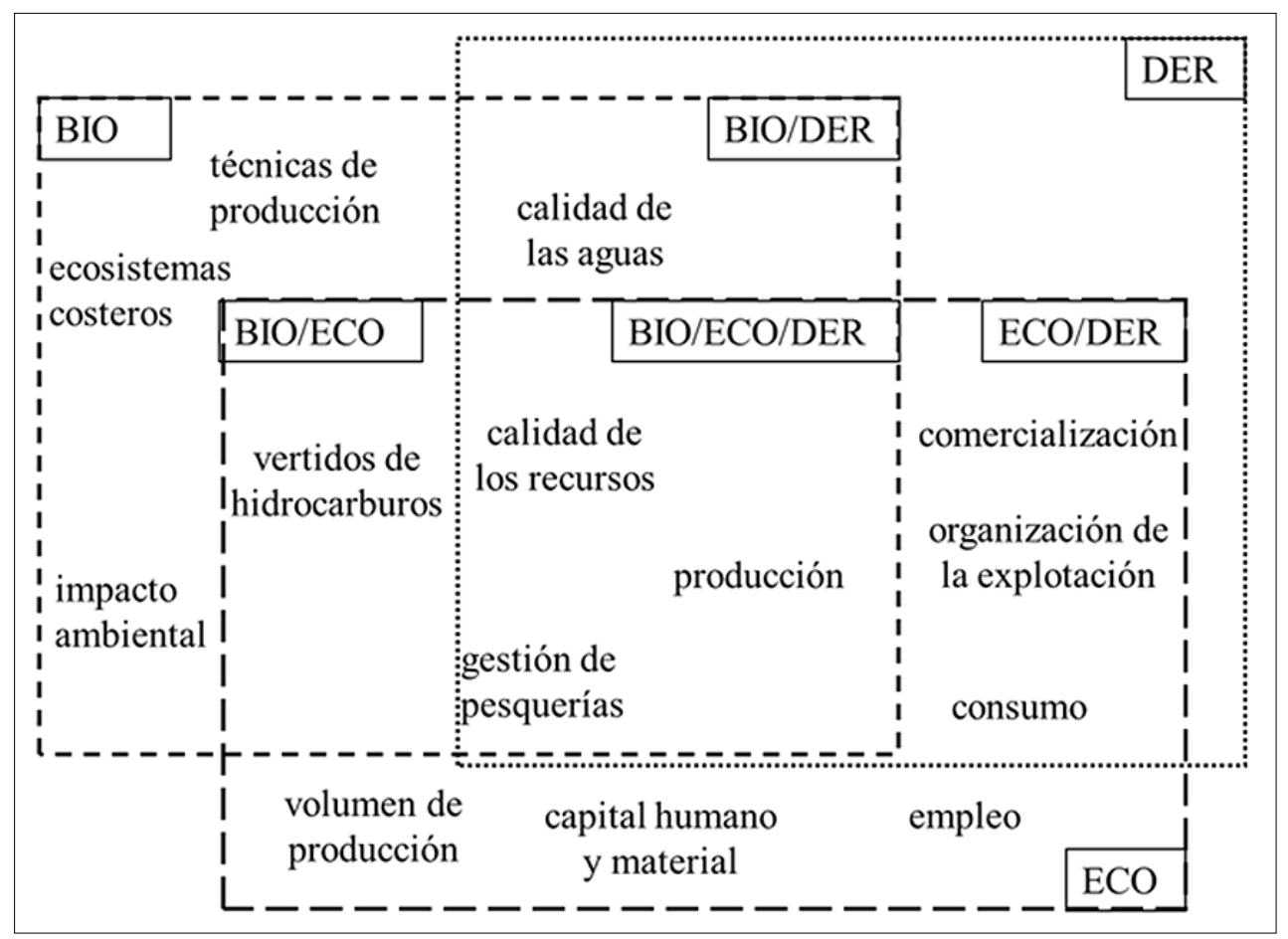

Figura 2. Temas de la actividad pesquera tratados por cada área disciplinar.

Sin embargo, temas como los vertidos marinos de hidrocarburos, por ejemplo, cuyas consecuencias ecológicas parecen prioritarias, también son abordados en relación con el impacto en la economía de las poblaciones costeras. Por otro lado, cuestiones relacionadas con la producción, comercialización, consumo o empleo son más recurrentes en los textos de economía, pero estos temas también son analizados desde el área de la biología o el derecho. Por último, no hemos identificado ningún tema que sea abordado con exclusividad en el en el subcorpus del derecho. Esto apunta a una cierta transversalidad de esta área, que será confirmada en los análisis posteriores.

En definitiva, la comparación entre textos de biología, economía y derecho nos muestra que, si bien hay temas periféricos que se asocian más a un área de especialidad en particular, existe un núcleo temático central presente en los tres subcorpus y que, eventualmente, cualquier aspecto de la pesca podría ser objeto de interés por parte de una determinada disciplina. 
En segundo lugar, hemos mirado qué tipo de conceptos, de los 95 analizados, estaban presentes en cada subcorpus.

\section{a) Subcorpus de biología}

En los textos de biología están representados lingüísticamente 73 conceptos. En la clase de humanos, tres conceptos correspondientes a grupos de profesionales (EMPRESA DE TRANSFORMACIÓN) no aparecen mencionados. Dentro de la clase de animales, no aparece el concepto de PESCA FRESCA, que representa el producto pesquero en una de sus modalidades de comercialización. En cuanto a la clase de actividades, algunas actividades de producción y transferencia, las que más relacionadas están con la faceta económica de la actividad pesquera, tampoco aparecen denominadas (ACUICULTURA DO RODABALLO). Sin embargo, tampoco figura el concepto de propiedad TALLA MíNIMA, que tiene una vinculación clara con la preservación de los recursos marinos, tema ampliamente tratado por los biólogos.

\section{b) Subcorpus de economía}

En el subcorpus de economía hemos encontrado representada casi la totalidad de los conceptos analizados (94). Esto se relaciona con el hecho de que la actividad pesquera es considerada ante todo una actividad productiva con importancia económica para las zonas geográficas de donde proceden los textos del corpus, a saber Galicia (España) y Francia. El único concepto sin representación lingüística es peso seco, una medida empleada en la investigación acuícola para evaluar la calidad o el crecimiento de los animales. Sin embargo, en los textos de economía también se incluyen conceptos vinculados con los aspectos medioambientales de la pesca, como CALIDAD DEL AGUA O IMPACTO ECOLÓGICO.

\section{c) Subcorpus de derecho}

En los textos de derecho aparecen representados lingüísticamente 73 conceptos. Encontramos conceptos típicamente biológicos, como PESO FRESCO, pero también conceptos relacionados con la comercialización de la pesca, como PRIMERA VENTA. Por otro lado, los conceptos más directamente vinculados con la legislación marítima, como las divisiones de las aguas (ZONA MARÍTIMO-TERRESTRE) también son tratados en los textos de las otras áreas. En este subcorpus se aprecia con claridad cómo los conceptos que conforman el sistema conceptual de la pesca, si bien se asocian típicamente a una determinada área disciplinar, pueden en realidad circular libremente entre las distintas áreas. En efecto, el derecho constituye un área de especialización transversal, que aborda todos los aspectos de la actividad pesquera desde la óptica específica de la legislación, ya sea la calidad de las aguas o la comercialización de los productos.

Tras analizar el solapamiento conceptual entre subcorpus diferenciados por áreas temáticas, las conclusiones que extraemos son similares a las del análisis temático. Si bien hay conceptos que son prototípicos o se abordan con más frecuencia desde una 
determinada área disciplinar, creemos que todos los conceptos de la pesca pueden ser tratados desde cualquier área y que, por tanto, no son exclusivos de un área específica.

\subsection{Solapamiento denominativo}

El siguiente paso en nuestro análisis ha consistido en comparar los términos empleados para hacer referencia a los mismos conceptos en los textos de biología, economía o derecho. A fin de comparar las elecciones denominativas, restringimos nuestras observaciones a los 62 conceptos con representación lingüística en los tres subcorpus:

Tabla 3. Dimensiones del corpus terminológico para el contraste entre áreas.

\begin{tabular}{|l|c|c|c|c|}
\cline { 2 - 5 } \multicolumn{1}{c|}{} & Biología & Economía & Derecho & Total \\
\hline $\mathrm{N}^{\circ}$ conceptos & 62 & 62 & 62 & 62 \\
\hline $\mathrm{N}^{o}$ variantes denominativas & 253 & 495 & 220 & 670 \\
\hline $\mathrm{N}^{o}$ contextos & 1.197 & 4.450 & 980 & 6.627 \\
\hline
\end{tabular}

En las Figuras 3-5 representamos gráficamente la proporción de términos compartidos entre las áreas disciplinares. Así, en la Figura 3 se puede apreciar que el subcorpus de biología posee el 35\% de variantes únicas, el 31\% compartidas con el subcorpus de economía y el 7\% con el de derecho, mientras que el 27\% de las variantes son comunes a las tres áreas:

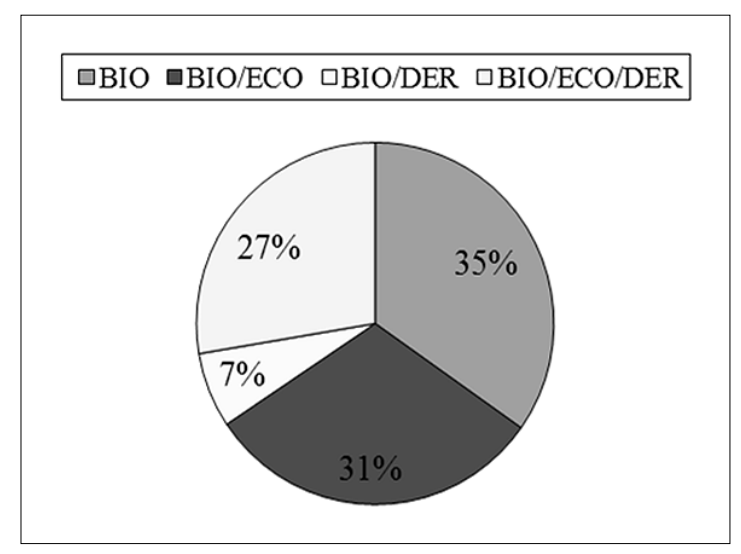

Figura 3. Proporción de términos del subcorpus de biología compartido con otras áreas.

En cuanto al subcorpus de economía (Figura 4), la mayor proporción de variantes son exclusivas de su área (57\%). El porcentaje compartido con la biología (16\%) o con el derecho (13\%) es muy similar, así como la proporción compartida por las tres subáreas (14\%). 


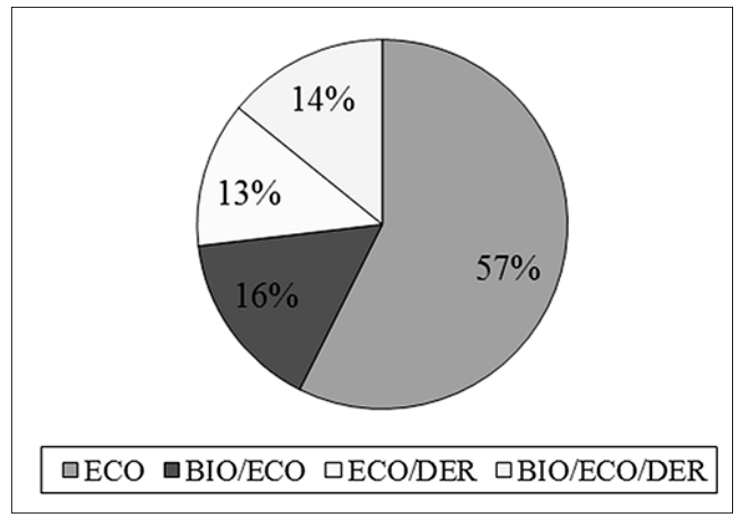

Figura 4. Proporción de términos del subcorpus de economía compartido con otras áreas.

Por último, el subcorpus de derecho (Figura 5) es el que presenta un menor número de variantes únicas $(32 \%)$ y el que posee la proporción más alta de variantes comunes a las tres áreas $(32 \%)$. Además, presenta una proporción mucho mayor de variantes compartidas exclusivamente con economía (28\%) que con biología (8\%).

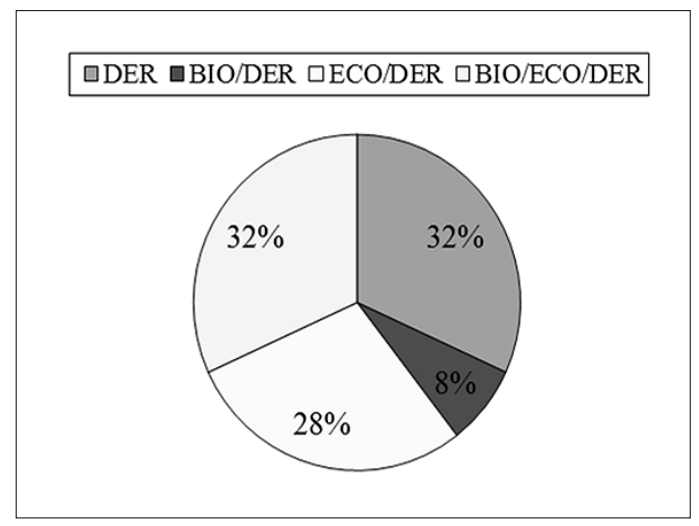

Figura 5. Proporción de términos del subcorpus de derecho compartido con otras áreas.

A la vista de los datos comparativos, podemos deducir que los subcorpus terminológicos de biología y economía son ligeramente más similares, y que el subcorpus de derecho muestra un comportamiento divergente. En cuanto al subcorpus de economía, el mayor porcentaje de variantes únicas se podría atribuir a que el tamaño del corpus es ligeramente mayor, pero creemos que también podría relacionarse con el hecho de que la pesca se conceptualiza, ante todo, como una actividad económica. En cuanto al comportamiento del subcorpus de derecho, se aprecia una mayor transversalidad que en las otras áreas, ya que figuran con más frecuencia términos empleados en economía o biología, y posee el menor número de elecciones denominativas únicas. 
A continuación, examinamos las elecciones denominativas comunes a los tres subcorpus y las elecciones exclusivas de cada área. Estos subconjuntos presentan unas diferencias importantes, las cuales indicamos a continuación:

\section{a) Variantes denominativas comunes}

En la Tabla 4 mostramos la distribución de las 70 variantes denominativas comunes a las tres subáreas en función del número de constituyentes y frecuencia de ocurrencia. Se destaca que la mayoría $(61,43 \%)$ son unidades monoléxicas ('pescador') o de dos constituyentes $(37,14 \%)$ y, como consecuencia de lo anterior, tienen una frecuencia de ocurrencia elevada, con una media de 41,46 ocurrencias por variante.

Tabla 4. Estructura y frecuencia media de las variantes comunes a los tres subcorpus.

\begin{tabular}{|c|l|l|l|l|}
\cline { 2 - 5 } \multicolumn{1}{c|}{} & \multicolumn{1}{c|}{ Monoléxico (\%) } & \multicolumn{1}{c|}{$\mathbf{2}$ const (\%) } & 3 const (\%) & \multicolumn{1}{c|}{ total (\%) } \\
\hline $\mathrm{N}^{\circ}$ variantes & $43(61,43 \%)$ & $26(37,14 \%)$ & $1(1,43 \%)$ & 70 \\
\hline Frecuencia media & 55,4 & 18,73 & 33 & 41,46 \\
\hline
\end{tabular}

Este subconjunto de denominaciones comunes está constituido por términos de la pesca extremadamente comunes, no marcados, correspondientes al nivel básico de categorización (Rosch, 1978) y que designan los conceptos más centrales del ámbito. Encontramos ocurrencias de denominaciones de profesionales 'pescador' (86) o conchyliculteur (51), las denominaciones de animales coquillage (38) o recurso (102), los lugares geográficos mer (123) o littoral (192) o las actividades marisqueo (110) o acuicultura (53).

Podemos decir que estas variantes comunes constituyen el núcleo terminológico de la pesca y son neutras en cuanto a la perspectiva, ya que no revelan ninguna conceptualización particular atribuible a una comunidad de expertos que se aproxima a la actividad pesquera desde su propia disciplina. Desde el punto de vista de la estabilidad denominativa, son los términos más fijados, menos dependientes del contexto de producción y los que aseguran una asociación referencial más duradera (Kleiber, 2001; Freixa, Fernández-Silva \& Cabré, 2008).

\section{b) Variantes denominativas exclusivas}

Si analizamos las variantes exclusivas de cada área en cuanto a la estructura morfosintáctica y frecuencia de ocurrencia (ver Tabla 5), se observa que la mayor proporción de variantes está constituida por términos de dos constituyentes $(58,4 \%$, 'estación de mostreo') y tres constituyentes (24,43\%, 'organismo de interés comercial'). Asimismo, se registra un número considerable de términos de estructura compleja, cuya longitud asciende hasta siete constituyentes ('zona de producción de moluscos bivalvos e outros invertebrados mariños vivos'). La frecuencia media de ocurrencia de este conjunto es mucho menor (3,49 ocurrencia/variante). 
Tabla 5. Estructura y frecuencia media de las variantes exclusivas a cada subcorpus.

\begin{tabular}{|l|l|c|c|c|c|c|}
\cline { 2 - 7 } \multicolumn{1}{l|}{} & Monoléxico(\%) & 2 const.(\%) & 3 const (\%) & 4 const. (\%) & $\mathbf{2}$ 5 const. (\%) & Total \\
\hline $\mathrm{N}^{\text {o variantes }}$ & $38(8,6)$ & $258(58,37)$ & $108(24,43)$ & $26(5,88)$ & $12(2,71)$ & 442 \\
\hline Fr. media & 13,58 & 2,96 & 1,84 & 1,19 & 2,58 & 3,49 \\
\hline
\end{tabular}

Por otro lado, las variantes monoléxicas $(8,6 \%)$, presentan una frecuencia media de ocurrencia mucho más baja que la que veíamos para las variantes comunes $(13,58$ frente al 55,4). Aunque encontramos unidades típicas de la terminología pesquera como pêcheur (32 ocurrencias) o aquaculture (17) hay muchos más términos genéricos -producteur, professionnel- o con configuraciones conceptuales menos frecuentes explotación directa de productos do mar - cuya asociación denominativa con el concepto es más ocasional, más dependiente del contexto o no tan prototípica de la terminología de la pesca.

En muchos casos, estas elecciones denominativas reflejan efectivamente una conceptualización particular asociada a una de las áreas. A continuación mostramos algunos ejemplos de variación donde se aprecia la perspectiva impuesta por el área disciplinar:

- Cultivo vs. producción

En la denominación de las actividades de cultivo, observamos una variación en cuanto a las preferencias denominativas de las distintas áreas. En los textos de economía, se emplea con mayor frecuencia la denominación genérica 'producción', que pone de relieve la finalidad económica de la actividad, mientras que en los textos de biología encontramos la denominación 'cultivo', que refleja la manera en que los moluscos son obtenidos. Véanse los siguientes contextos del concepto SISTEMA DE CULTiVo, en el que se ven las diferencias de perspectiva entre la biología y la economía:

...O ** sistema de cultivo** escollido foi o diseñado e patentado pola empresa de Rianxo SERVIMAR NORTE SL [...]. Consiste nuha serie de bandexas apilables recubertas cun tubo de rede intercambiable por outros con diferente luz de malla...

...Integración de **sistemas de producción** en policultivo (cultivos 23870/ integrados de varias especies), co que se maximiza a utilización da ECO enerxía e do espazo, redúcense os impactos e increméntase a productividade...

- Barco de pesca/pesqueiro vs. unidade de producción/unidade produtiva

En la denominación del concepto EMBARCACIÓN encontramos, en el subcorpus de biología, las variantes únicas 'barco de pesca' y pesqueiro, en el que se identifica el objeto por la actividad específica: 
...Orde do 24 de febreiro de 2003 pola que se establecen medidas 8822/ BIO específicas para compensar determinados danos producidos nos **barcos de pesca** e auxiliares de acuicultura como consecuencia dos vertidos de fuelóleo do petroleiro Prestige...

...Para a situación dos lugares de mostreo empregáronse os datos dou-

9025/ BIO tras investigacións realizadas polos [...]** pesqueiros** empregados nas prospeccións anteriores, tanto arrastreiros como de artes fixas...

En los textos de economía encontramos la misma configuración conceptual (batean de pêche), pero también denominaciones como unidade de producción, en que el barco es conceptualizado como un elemento implicado en la producción industrial:

...A pesca de baixura/litoral inclue o conxunto de **unidades de pro-

8775/ ECO dución** que faenan dentro dos límites das rías e no litoral acantilado, captura espécies próprias das rías para venda só en fresco, ao dia...

- Site d'élevage/zona productiva vs. zona de producción de moluscos bivalvos e outros invertebrados mariños vivos

En las variantes del concepto ZONA DE PRODUCCIÓN que incluimos en este ejemplo se aprecia una característica específica de la denominación en los textos de derecho, a saber, el empleo de sintagmas terminológicos extensos como 'zona de producción de moluscos bivalvos' e outros invertebrados mariños vivos.

...Orde do 23 de novembro do 2000, corrixida pola Orde de 19 de DER de moluscos bivalvos e outros invertebrados mariños vivos** no litoral español...

El uso de estas unidades se justifica, en el marco legislativo, por la necesidad de especificar exactamente qué tipo de entidades están incluidas en la extensión del concepto. Por ello no es extraño encontrar denominaciones extensas que se asemejan a definiciones del concepto (de las 12 variantes únicas con 5 o más constituyentes, 11 pertenecen al subcorpus de derecho). En otras áreas, en cambio, la finalidad de la denominación consiste en identificar de manera eficaz y rápida el concepto mediante una opción lingüísticamente más económica:

...Cette première année d'étude a permis de mettre en évidence des

11202/ BIO différences significatives entre les **sites d'élevage** d'un point de vue croissance, composition de la chair... 


\subsection{Perspectivas de conceptualización de los conceptos de animales según área disciplinar}

Como última etapa en nuestra investigación, comparamos cuantitativamente las elecciones denominativas referidas a conceptos de animales. Hemos seleccionado esta clase conceptual porque en ella conviven dos perspectivas de conceptualización en el marco de la actividad pesquera:

- La perspectiva 'perceptiva', en la que el animal se conceptualiza como un ser vivo y se caracteriza a partir de su morfología (anatomía) o su conducta (etología).

- La perspectiva 'funcional', en la que se conceptualiza según su función para el ser humano en el marco de la actividad pesquera, ya sea como un recurso natural explotable o como un producto resultante de dicha actividad.

Para comparar las diferencias denominativas entre áreas, observaremos la distribución de los patrones denominativos en los tres subcorpus. Los datos corresponden a 12 conceptos de animales ( 7 tipos de animales y 5 grupos de animales), 131 variantes denominativas y 1.225 contextos.

En la Tabla 6 describimos los patrones denominativos de clase de animales y en las Figuras 6-7 comparamos la productividad en cada área disciplinar, en cuanto a la generación de términos (Figura 6) y en cuanto a su frecuencia de ocurrencia en cada subcorpus (Figura 7). Los patrones 1, 2 y 3 reflejan la perspectiva perceptiva y los patrones 4, 5 y 6 la perspectiva funcional.

Tabla 6. Patrones denominativos de la clase de animales.

\begin{tabular}{|c|c|c|c|c|c|}
\hline \multirow{2}{*}{\multicolumn{2}{|c|}{ Perspectiva }} & \multicolumn{3}{|c|}{ Patrón denominativo } & \multirow[b]{2}{*}{ Ejemplos } \\
\hline & & \multirow{2}{*}{\begin{tabular}{|l|} 
Núcleo \\
ANIMAL
\end{tabular}} & \multirow{2}{*}{ Relación } & \multirow{2}{*}{ Modificador } & \\
\hline \multirow{4}{*}{$\begin{array}{l}\text { PERCEP } \\
\text { TIVA }\end{array}$} & 1 & & & & ostra \\
\hline & 2 & $\begin{array}{l}\text { ANIMAL/OB- } \\
\text { JETO }\end{array}$ & $\begin{array}{l}\text { concepto } \\
\text { atribuido }\end{array}$ & ANIMAL $(+$ ANIMAL + ANIMAL $)$ & comunidade biolóxica \\
\hline & \multirow[t]{2}{*}{3} & \multirow{2}{*}{ ANIMAL } & $\begin{array}{l}\text { nat.interna/ } \\
\text { forma }\end{array}$ & PROPIEDAD $(+$ ACTIVIDAD $)$ & mollusco bivalvo \\
\hline & & & localización & LUGAR & organisme marin \\
\hline \multirow{5}{*}{$\begin{array}{l}\text { FUNCIO } \\
\text { NAL }\end{array}$} & 4 & OBJETO & & & producto \\
\hline & \multirow{3}{*}{5} & \multirow{3}{*}{$\begin{array}{l}\text { ANIMAL/ } \\
\text { OBJETO }\end{array}$} & uso/función & OBJETO/ACTIV./ARTEFACTO & aproveitamento pesqueiro \\
\hline & & & localización & LUGAR & recurso mariño \\
\hline & & & $\begin{array}{l}\text { estado/nat. } \\
\text { externa/rol }\end{array}$ & ENT. ABSTRACTA/PROPIEDAD & especie de interese comercial \\
\hline & 6 & OBJETO & $\begin{array}{l}\text { origen/loc./ } \\
\text { nat. ext./rol }\end{array}$ & $\begin{array}{l}\text { ACTIVIDAD/LUGAR/ } \\
\text { PROPIEDAD }\end{array}$ & alimento do mar \\
\hline
\end{tabular}


- El patrón 1, monoléxico, selecciona conceptos de animales en la forma denominativa. Estas denominaciones reflejan criterios de clasificación propios de las taxonomías biológicas: la estructura interna (organismo), la especie (ostra), la característica externa (bivalvo) o interna (molusco), el hábitat común (poboación), etc. Es un patrón poco productivo a la hora de formar términos pero muy frecuente en contexto, debido a su carácter monoléxico. $\mathrm{Su}$ frecuencia de ocurrencia es mayor en el corpus de biología $(34,5 \%$ del total de ocurrencias) que en el de economía $(26,6 \%)$ o derecho $(11,2 \%)$.

- El patrón 2 se emplea mayoritariamente en conceptos de grupos de animales. En el núcleo se seleccionan conceptos de grupos de animales, y como característica distintiva, en el modificador, aparecen el(los) tipo(s) de animal(es) que los integran (comunidade biolóxica, huître Crassostrea Gigas, recurso peixe, crustáceos e moluscos). La productividad de este patrón en nuestro corpus es limitada, ya que en las denominaciones de conceptos de grupos de animales predomina la conceptualización como objeto (patrones 5 y 6 ). No obstante, es ligeramente superior en el corpus de biología (2,6\% de las ocurrencias) que en el de economía $(0,4)$, mientras que está ausente en el de derecho.

- El patrón 3 selecciona conceptos de animales en el núcleo, mientras que los modificadores especifican propiedades de naturaleza (poboación natural), de forma (molusco bivalvo) y, en algunos casos, se añade una acción característica del animal (mollusque lamellibranche filtreur). También encontramos la especificación del hábitat del animal (organisme marin). Su productividad en la formación de variantes no es muy elevada, pero es el patrón poliléxico con la mayor frecuencia de ocurrencia en corpus, especialmente en los subcorpus de biología $(28,6 \%)$ y derecho $(27,8 \%)$.

- El patrón 4 representa a los animales como objetos dentro de la actividad pesquera, ya como recursos explotables a través de esta (ressource) o como productos de la actividad (producto). Su presencia es inferior al patrón monoléxico que refleja la perspectiva biológica en los corpus de biología $(10,1 \%$ ) y economía (4,1\%), y superior en el corpus de derecho $(12,1 \%)$.

- El patrón 5 conceptualiza el animal como recurso explotable, situándolo con anterioridad a la realización de la actividad pesquera: en el núcleo se alternan conceptos de animales y de objetos concebidos en su utilidad para el hombre (fauna piscicola e marisqueira, stock halieutique). En los modificadores, las dimensiones relevantes vinculan a los animales con los diferentes aspectos de la actividad pesquera: las actividades realizadas sobre ellos (aproveitamento pesqueiro), los lugares (recurso mariño), los medios de producción (mexillón de batea) o la función para el ser humano en el marco de la actividad pesquera (especie de interese comercial). Es el patrón más productivo en cuanto a la generación de términos en los textos de biología (38\% de las variantes lo seleccionan) y economía (39,6\% de las variantes), si bien también es el que mayor diversidad interna presenta. Su presencia no es tan marcada en los textos de derecho (13,9\% variantes). 
- El patrón 6 sitúa al animal después de la realización de la actividad productiva. Los núcleos ya no son animales o recursos, sino productos resultantes de la actividad productiva (producto). En consecuencia, los modificadores especifican el origen del producto (producto da pesca), su procedencia (alimento do mar) y/o alguna propiedad de estado (producto da pesca en fresco). Este patrón ocupa el primer lugar en productividad para el subcorpus de derecho $(38,9 \%$ ) y el segundo lugar en economía $(26 \%)$, mientras que su presencia en los textos de biología es inferior (7,9\% de las variantes).

En la Figura 6 representamos gráficamente la productividad de los distintos patrones en la formación de variantes denominativas, y en la Figura 7 su productividad en cuanto a la frecuencia de ocurrencia en corpus.

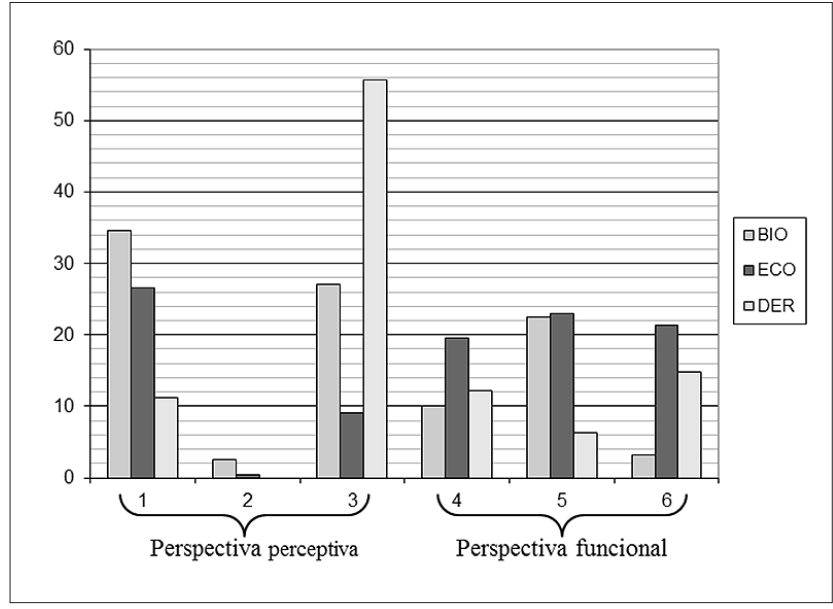

Figura 6. Productividad en la formación de variantes.

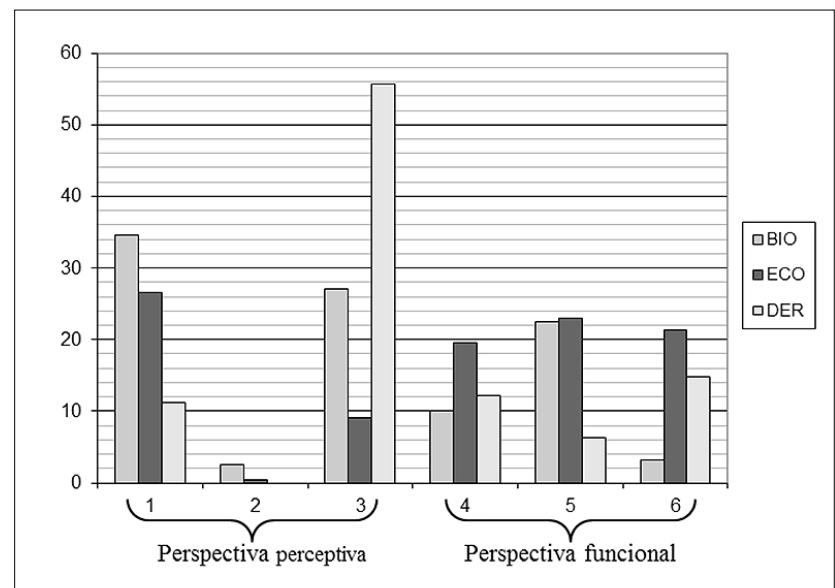

Figura 7. Productividad en corpus.

Nuevamente se observa que los patrones 'perceptivos' son más frecuentes en biología que en economía o derecho. Del mismo modo, la perspectiva 'funcional' es la más frecuente 
en el subcorpus de economía, tanto en número de variantes $(69,8 \%)$ como en frecuencia de uso (51,6\%). En cuanto al área del derecho, una ligera mayoría de variantes reflejan la perspectiva funcional $(53,3 \%)$, pero esta preferencia se invierte cuando examinamos la frecuencia de uso $(33,2 \%)$. La particularidad denominativa asociada a esta área no es tanto la preferencia hacia una determinada perspectiva como la elevada productividad de los patrones poliléxicos en contraste con los monoléxicos. En ambos gráficos se observa que es el área en la que los patrones monoléxicos (1 y 4) tienen menor importancia.

\section{CONCLUSIONES}

En este artículo nos hemos interesado por la manera en que se construye y comparte el conocimiento en un ámbito interdisciplinar, y de qué manera se refleja la multiplicidad de perspectivas disciplinares en el uso de la terminología. Para ello, en un corpus de textos sobre pesca, comparamos los temas, los conceptos, y las variantes denominativas empleadas por especialistas de distintas disciplinas.

En primer lugar, observamos una elevada coincidencia en los temas y conceptos abordados por los distintos colectivos. Si bien se encontraron temáticas más naturalmente vinculadas a una determinada área disciplinar, se observó la presencia de un núcleo temático y conceptual común, en el que se incluyen la mayoría de conceptos analizados. De las tres áreas, la de derecho es la que mayor transversalidad presentó, ya que en sus textos se abordan temas y conceptos típicos del área de economía o biología. Nuestras observaciones sugieren el carácter interdisciplinar de este ámbito, y su construcción colectiva a partir del aporte de varias disciplinas, así como la libertad de circulación de los conceptos entre áreas disciplinares.

En cuanto al uso de la terminología, observamos diferencias entre la terminología compartida por los tres colectivos y la terminología empleada exclusivamente en cada área. El núcleo terminológico común está constituido por términos más cortos (fundamentalmente monoléxicos), frecuentes, centrales en la terminología pesquera, neutros desde el punto de vista de la perspectiva y con un vínculo más estable con el referente. Por el contrario, los términos no compartidos son más extensos, más inestables, más dependientes del contexto y, en ocasiones, reflejan perspectivas de conceptualización particulares, no prototípicas del ámbito de la pesca.

En tercer lugar, investigamos si las denominaciones de los conceptos de animales reflejan una perspectiva de conceptualización propia del área disciplinar y en qué medida difieren con respecto a las visiones del concepto reflejadas en otras áreas. Los resultados mostraron que existen dimensiones conceptuales cuya prominencia está por encima de las diferencias entre áreas temáticas, pero que existe una parcela denominativa en la que se manifiesta la perspectiva de conceptualización propia de cada área.

Estos resultados, a nuestro juicio, están en consonancia con una visión flexible y dinámica de los procesos de categorización y denominación del conocimiento 
especializado. En nuestro análisis hemos visto cómo confluyen una multiplicidad de perspectivas en la conceptualización y en la denominación de los mismos hechos. Si bien el área disciplinar impone una determinada perspectiva de conceptualización sobre la realidad que se refleja en la terminología empleada, esto no impide que las distintas disciplinas intercambien conceptualizaciones, puntos de vista o terminología, y que expertos de un área comprendan y reutilicen términos de otras áreas. En este sentido, la variación terminológica se relaciona estrechamente con la variabilidad de los procesos de categorización y estructuración del conocimiento especializado. Consideramos que este fenómeno no puede ser interpretado únicamente como un descuido o un recurso estilístico cuyo único fin es evitar la repetición, sino que adquiere una función cognitiva de primer orden. Mediante la variación terminológica se hace referencia a un mismo concepto a través de variantes que enfatizan diferentes aspectos de su contenido, que muestran diferentes perspectivas sobre los mismos hechos, proporcionando una visión más rica y flexible del concepto. Consideramos que en un contexto interdisciplinar, la variación terminológica desempeña un papel importante en el diálogo entre disciplinas, pues es a través de variantes que reflejan perspectivas diferentes que se comparten distintas visiones sobre los mismos conceptos, contribuyendo a la construcción colectiva del conocimiento. 


\section{REFERENCIAS BIBLIOGRÁFICAS}

Bertaccini, F. \& Matteucci, A. (2005). L'approche variationniste à la pratique terminologique d'entreprise. Meta, 50(4). [CD-ROM]

Bouveret, M. \& Gaudin, F. (1997). Du flou dans les catégorisations: Le cas de la bioinformatique. En C. De Schaetzen (Ed.), Actes des Troisièmes journées internationales de terminologie, Bruxelles 31 mai-1er juin 1996 (pp. 63-72). Louvain la Neuve: Peeters.

Bowker, L. (1997). You say "flatbed colour scanner”, I say “colour flatbed scanner”: A descriptive study of the influence of multidimensionality on term formation and use with special reference to the subject field of optical scanning technology. Terminology, 4(2) 275-302.

Bowker, L. \& Hawkins, S. (2006). Variation in the organization of medical terms: Exploring some motivations for term choice. Terminology, 12(1) 79-110.

Bunge, M. A. (1966). La ciencia: Su método y su filosofía. Buenos Aires: Siglo Veinte.

Cabré, M. T. (1999a). La terminología: Representación y comunicación: Elementos para una teoría de base comunicativa y otros artículos. Barcelona: IULA-UPF.

Cabré, M. T. (1999b). El discurs especialitzat o la variació funcional determinada per la temàtica: Noves perspectives. En T. Cabré (Ed.), La terminología. Representación y comunicación. Una teoría de base comunicativa y otros articulos (pp. 151-173). Barcelona: IULA.

Cabré, M. T. (2008). El principio de poliedricidad: La articulación de lo discursivo, lo cognitivo y lo lingüístico en Terminología (I). IBÉRICA, 16, 9-36.

Cabré, M. T. \& Estopà, R. (2005). Unidades de conocimiento especializado: Caracterización y tipología. En T. Cabré \& C. Bach (Eds.), Coneixement, llenguatge $i$ discurs especialitzat (pp. 69-93). Barcelona: IULA, Documenta Universitaria.

Chalmers, A. (1990). Science and its fabrication. Buckingham: Open University Press.

Collet, T. (2004). What's a term? An attempt to define the term within the theoretical framework of text linguistics. Linguistica Antverpiensia, 3, 99-111.

Conceição, M. C. (2006). Há mar e mar... Há termos e termos. Quando os termos dizem mas não condizem! En T. Cabré, R. Estopà \& C. Tebé (Eds.), Actas del IX Simposio Iberoamericano de terminología RITERM04 (pp. 495-502). Barcelona: IULA, Documenta Universitaria.

Diki-Kidiri, M. (2008). Le vocabulaire scientifique dans les langues africaines: Pour une approche culturelle de la terminologie. Paris: Karthala.

Faber, P. (Ed.). (2012). A cognitive linguistics view of terminology and specialized language. Berlin/ Boston: De Gruyter Mouton. 
Fernández-Silva, S. (2011). Variación terminológica y cognición: Factores cognitivos en la denominación del concepto especializado. Barcelona: Tesis en Xarxa.

Fernández-Silva, S. (2013). Variación denominativa y punto de vista. Revista Debate Terminológico, 9, 11-37.

Fernández-Silva, S., Freixa, J. \& Cabré, M. T. (2011). A proposed method for analysing the dynamics of cognition through term variation. Terminology, 17(1), 49-73.

Fernández-Silva, S., Cabré, M. T. \& Freixa, J. (2012). A cognitive approach to synonymy in terminology. En M. Brdar, I. Raffaelli \& M. Žic Fuchs (Eds.), Cognitive Linguistics between Universality and Variation (pp. 189-212). Newcastle: Cambridge Scholars Publishing.

Freixa, J. (2002). La variació terminològica: Anälisi de la variació denominativa en textos de diferent grau d'especialitració de l'àrea de medi ambient. Barcelona: IULA-UPF.

Freixa, J. (2006). Causes of denominative variation in terminology: A typology proposal. Terminology, 12(1), 51-77.

Freixa, J., Fernández-Silva, S. \& Cabré, T. (2008). La multiplicité des chemins dénominatifs. Meta, 53(4), 731-747.

Gambier, Y. (1991). Presuposés de la terminologie: Vers une remise en cause. Cabiers de linguistique sociale, 18, 31-58.

Gaudin, F. (2003). Socioterminologie: Une approche sociolingnistique de la terminologie. Bruselas: De Boek Duculot.

Halliday, M. \& Hasan, R. (1976). Cohesion in English. Londres: Longman.

Hyland, K. (2000). Disciplinary discourses: Social interactions in academic writing. Londres: Longman.

Hyland, K. \& Bondi, M. (2006). Academic discourse across disciplines. Bern: Peter Lang.

Ibáñez, R. (2010). The disciplinary text genre as a means for accessing disciplinary knowledge: A study from genre analysis perspective. En G. Parodi (Ed.), Academic and professional discourse genres in Spanish (pp. 189-112). Amsterdam: John Benjamins.

Kageura, K. (2002). The dynamics of terminology: A descriptive theory of term formation and terminological growth. Amsterdam: John Benjamins.

Kleiber, G. (2001). Remarques sur la dénomination. Cabiers de praxématique, 36, 21-41.

Kocourek, R. (1991). La langue française de la technique et de la science: Vers une linguistique de la langue savante. Wiesbaden: Brandstetter. 
Louwerse, M. M. (2004). Un modelo conciso de cohesión en el texto y coherencia en la comprensión. Revista Signos. Estudios de Lingüistica, 37(56), 41-58.

Névéol, A. \& Ozdowska, S. (2006). Terminologie médicale bilingue anglais/français: Usages clinique et législatif. Glottopol, 8, 5-21.

Parodi, G. (2010a). Academic and professional genres: Variation across four disciplines. En G. Parodi (Ed.), Academic and professional discourse genres in Spanish (pp. 65-82). Amsterdam: John Benjamins.

Parodi, G. (2010b). The textbook genre and its rethorical organisation in four scientific disciplines: Between abstraction and concretness. En G. Parodi (Ed.), Academic and professional discourse genres in Spanish (pp. 171-188). Amsterdam: John Benjamins.

Rosch, E. (1978). Principles of categorization. En E. Rosch \& B. Lloyd (Eds.), Cognition and Categorization (pp. 27-48). Hillsdale: Erlbaum.

Sabaj, O. (2012). Uso de movidas retóricas y patrones léxico-gramaticales en artículos de investigación en español. Implicancias para la enseñanza de la escritura científica. Boletín de filología, 47(1), 165-186.

Samraj, B. (2005). An exploration of a genre set: Research article abstracts and introductions in two disciplines. English for specific purposes, 24(2), 141-156.

Samraj, B. (2008). A discourse analysis of master's theses across disciplines with a focus on introductions. Journal of English for academic purposes, 7(1), 55-67.

Seghezzi, N. (2011). Variación terminológica y canal de comunicación. Estudio contrastivo de textos especializados escritos y orales sobre lingüistica. Barcelona: Tesis en Xarxa.

Suárez, M. (2004). Análisis contrastivo de la variación denominativa en textos especializados: Del texto original al texto meta. Barcelona: IULA-UPF.

Swales, J. (1990). Genre analysis: English in academic and research settings. Cambridge: Cambridge University Press.

Tebé, C. (2005). La representació conceptual en terminologia. L'atribució temàtica en els bancs de dades terminologiques. Barcelona: IULA-UPF.

Temmerman, R. (2000). Towards new ways of terminology description: The sociocognitive-approach. Amsterdam: John Benjamins.

Wüster, E. (1979). Einführung in die Allgemeine Terminologielehre und Terminologische Lexikographie. Viena: Springer. [Traducción castellana: 1998. Introducción a la teoría general de la terminología y a la lexicografía terminológica. En T. Cabré (Ed.) Barcelona: IULA-UPF. 


\section{AGRADECIMIENTOS}

* Para los datos en gallego hemos contado con la ayuda de Lino Lema Bouzas, Director General de Investigación y Desarrollo Pesquero de la Consellería de Pesca y Asuntos Marítimos de la Xunta de Galicia. Nuestros colaboradores para el francés son Patricia Priour, doctora en ecología marina, y Daniel Priour, ingeniero especializado en tecnologías marinas para la pesca del IFREMER (Institut de Recherche pour le Développement de la Mer). Por último, para la equivalencia entre lenguas hemos contado con la colaboración de Antonio Gutiérrez González, de la dirección General de Pesca de la Comisión Europea. A todos ellos queremos hacer constar nuestro más sincero agradecimiento por su valiosa colaboración. 